Horizons philosophiques

\title{
Entre philosopher et présenter l'histoire de la Philosophie : un juste milieu dans le dialogue
}

\section{Marie-Georges Bélanger}

Volume 11, numéro 1, automne 2000

L’amodernité de la photographie?

URI : https://id.erudit.org/iderudit/802955ar

DOI : https://doi.org/10.7202/802955ar

Aller au sommaire du numéro

Éditeur(s)

Collège Édouard-Montpetit

ISSN

1181-9227 (imprimé)

1920-2954 (numérique)

Découvrir la revue

Citer cet article

Bélanger, M.-G. (2000). Entre philosopher et présenter l'histoire de la

Philosophie : un juste milieu dans le dialogue. Horizons philosophiques, 11(1),

135-144. https://doi.org/10.7202/802955ar d'utilisation que vous pouvez consulter en ligne.

https://apropos.erudit.org/fr/usagers/politique-dutilisation/ 


\section{ENTRE PHILOSOPHER ET PRÉSENTER L'HISTOIRE DE DE LA PHILOSOPHIE : UN JUSTE MILIEU DANS LE DIALOGUE1}

Comment se porte la philosophie au collégial? Au cours de mes études collégiales, j'ai pu constater que l'opinion générale des élèves était négative à l'égard de la philosophie. Selon moi, la réforme n'a rien changé à cette situation, si ce n'est qu'elle a fait plaisir aux malheureux élèves en enlevant un cours obligatoire. Le problème est particulier à cette matière principalement parce qu'elle est totalement méconnue des élèves avant leurs études collégiales. La question que j'aimerais traiter concerne l'étiologie du problème : est-ce vraiment la philosophie qui fait défaut ou plutôt son rapport à l'enseignement? C'est sur ce lien entre matière et pédagogie que je souhaite m'attarder ici. II s'agit certes d'un problème actuel pour les futurs professeurs de philosophie au collégial, puisque nous en sommes à nous demander, nous bacheliers en philosophie, si nous devons suivre des cours de pédagogie de l'enseignement au collégial ou si nous devons plutôt nous spécialiser en philosophie pour ensuite aller directement dans les salles de classes. Dans le but de susciter le dialogue entre les professeurs actuels et futurs, je tenterai d'abord de cerner ce qui m'apparaît être la cause du problème des cours de philosophie au collégial, pour ensuite proposer une solution concrète qui repose sur la méthode d'enseignement, et non sur le contenu. $\mathrm{Si}$, comme nous en ferons l'hypothèse, la philosophie est autant une histoire qu'un savoir-faire, nous tenterons de découvrir le rôle que la méthode de Philosophie pour les enfants, appliquée au niveau collégial québécois, peut jouer pour permettre de rejoindre ces deux composantes indissociables.

\section{Qu'est-ce que la philosophie?}

Je formule l'hypothèse que la philosophie est un dialogue. Pour appuyer mes propos, je me réfère à la méthode de Socrate et aux textes de Platon. Socrate se promenait dans la cité en quête d'interlocuteurs pour philosopher avec eux. Ce philosophe n'a jamais

1. Une première version de cet article a fait l'objet d'une communication présentée le 14 juin 1999, au département de philosophie de l'Université de Montréal, dans le cadre d'un colloque intitulé "L'enseignement de la philosophie". 
écrit de traités, il pratiquait la philosophie. Nous pouvons dire que pour lui, la philosophie se vivait dans le dialogue. Platon aussi redoutait les écrits; il aurait souhaité qu'on se limite à l'enseignement oral. De plus, sa manière d'écrire illustre ce qu'est originairement la philosophie : un dialogue. Aujourd'hui cependant, il me semble que l'enseignement de la philosophie privilégie davantage l'étude de textes et de systèmes qu'une pratique dialogique. Ces philosophes nous invitent pourtant, par leur méthode, à participer à la vie philosophique, plutôt qu'à étudier leurs écrits dogmatiquement. Ainsi, puisque nous supposons qu'à l'origine, la philosophie était dialogue, alors il semble que pour être en mesure de faire comprendre ce qu'est la philosophie aux élèves du collégial, il faudrait commencer par les amener à dialoguer. Or, le début du dialogue, c'est l'interrogation. Pour recréer le dialogue originel, et mettre les collégiens en contact avec la philosophie, je crois que le meilleur moyen est de permettre aux élèves de s'approprier les questions philosophiques. Ceci équivaut à leur demander ce qu'ils répondraient aux questions que les philosophes se sont posées. Ensuite, il est pertinent de leur présenter les réponses auxquelles les penseurs ont été conduits, mais seulement, il me semble, après qu'ils se soient approprié le questionnement. Par exemple, en demandant aux élèves ce qu'ils pensent de l'amour (à l'intérieur d'un cadre de recherche bien défini), on peut leur faire apprécier davantage le discours de Platon dans $L e$ Banquet, qui leur semblera déjà un peu moins déconnecté de leur expérience. Avant d'avancer plus loin, je souligne que je n'entends pas remplacer tous les cours de philosophie par des moments de discussions fertiles entre les élèves. Ce que je propose est plutôt une méthode systématique complémentaire qui pourrait permettre de faire émerger un enseignement qui m'apparaîtrait désormais complet puisqu'il engloberait les deux composantes de la philosophie que je privilégie ici : le savoir-faire et l'histoire.

Le dialogue est-il déjà présent dans les cours de philosophie du collégial? Chez les professeurs, on note deux grandes tendances : enseigner l'histoire de la philosophie, et pratiquer la philosophie en apprenant aux élèves à penser par eux-mêmes. Une des difficultés pédagogiques concerne la gestion de ces deux extrêmes. Pour ce qui est de l'histoire de la philosophie, n'importe quel diplômé peut l'enseigner, par des leçons magistrales, et n'importe quel élève "doué en par cœur" peut la maîtriser. Mais philosopher, c'est un art. Philosopher implique l'utilisation d'une pensée critique. Les élèves, et les citoyens de la société en général, peuvent avoir de la difficulté à user de jugement s'ils n'ont jamais acquis les outils de la pensée 
qui permettent de développer un esprit critique. Nous disions que le commencement du dialogue, c'est l'interrogation. II ne suffit cependant pas de se questionner pour philosopher; le questionnement n'est que le point de départ de la philosophie. Ensuite, pour passer des questions au dialogue, la tâche n'est pas mince. Afin de parvenir à penser librement, il faut acquérir les outils de la pensée, que sont les exemples, contre-exemples, hypothèses, conséquences, présupposés, et bien d'autres. Et comme il ne suffit pas de savoir lire une partition pour savoir jouer du piano, il ne suffit pas de simplement connaître ces outils pour savoir penser par soi-même. II faut de la pratique et un encadrement qui facilite l'acquisition des outils de la pensée. Les deux aspects de la philosophie, les problématiques constituant son histoire et l'art de philosopher, sont selon moi les deux constituants d'une bonne culture philosophique, à condition qu'on les maîtrise. Je ne crois pas que l'on doive choisir entre enseigner aux élèves la philosophie et leur apprendre à philosopher. Je crois au contraire, comme je le démontrerai dans la prochaine section, qu'une bonne formation doit reposer sur les deux éléments; c'est pour cette raison que nous devrions trouver un moyen de les faire se rencontrer. Or, le point de rencontre originel est le dialogue philosophique. Alors l'enseignement de l'histoire de la philosophie devrait lui aussi être un dialogue s'il veut rester fidèle à sa matière. Un philosophe américain, Matthew Lipman, affirme dans ce sens qu'on ne peut enseigner correctement la philosophie sans dans une certaine mesure faire soi-même de la philosophie et initier une activité similaire chez l'élève. Le contenu de la philosophie serait ainsi difficilement séparable de sa méthodologie. Si c'est le cas, l'enseignement de la philosophie devrait alors être lui aussi, comme sa matière, dialogique. C'est ainsi que nous sommes amenés à examiner la méthode de Philosophie pour les enfants.

\section{La philosophie pour les enfants}

Je fais un bref rappel historique afin de montrer d'où émerge le principe de la Philosophie pour les enfants, programme qui a vu le jour aux États-Unis dans les années 70 . Un professeur universitaire, Matthew Lipman, a constaté avec désarroi que ses étudiants, à qui il enseignait la logique, ne savaient pas penser par et pour eux-mêmes. II a donc mis sur pied une approche qui permettait à ses élèves de développer des outils qui leur donnaient accès à une pensée structurée. II s'est cependant rendu compte que le problème, pour être réglé efficacement, devait être abordé dès les premières années 
de l'éducation des enfants. Sa solution fut le programme de Philosophie pour les enfants. II a écrit des romans - indiqués dans notre bibliographie - dont les héros étaient des jeunes élèves du primaire, et il y a subtilement inséré des problèmes philosophiques. Ainsi, après la lecture en commun de tels textes, les enfants sont susceptibles d'avoir quelques interrogations sur le contenu. C'est à partir de ce questionnement propre à l'enfant que les discussions ont lieu. Le rôle de l'animateur est de s'assurer que la discussion soit philosophique, et non psychologique par exemple. Pour atteindre ce but, il doit insister sur l'utilisation d'outils de la pensée tels que la formulation d'hypothèses, d'exemples, de contre-exemples, de définitions, de présupposés, de critères, de comparaisons, et de conséquences. Les avantages que retirent les élèves concernent autant les relations humaines que les compétences intellectuelles. En effet, le dialogue développe chez l'élève le respect, l'écoute, l'entraide, l'observation et la collaboration, et lui permet de développer les habiletés à raisonner, à rechercher et à organiser l'information. Aux fondements d'une telle communauté de recherche, il y a donc une structure et un ensemble d'outils et de moyens qui permettent d'atteindre des objectifs précis.

Pour s'assurer d'un réel apprentissage de la part des élèves, l'élément essentiel reste, bien sûr, le support du professeur (transformé en animateur). Le programme de Philosophie pour les enfants a prévu un matériel d'accompagnement pour les animateurs de discussions. D'une part, les romans insérés dans le programme de Lipman établissent le lien entre la philosophie et l'expérience des élèves, suscitant leur intérêt pour le questionnement philosophique. D'autre part, les plans de discussions auquel l'animateur peut se référer sont des questions, issues de l'histoire de la philosophie et inspirées des romans philosophiques, qui suscitent le dialogue. Ces plans de discussions contribuent à conserver la rigueur philosophique dans le dialogue. Idéalement, pour permettre l'union du dialogue et de I'histoire de la philosophie, l'animateur doit toujours avoir en tête les grandes lignes de l'histoire de la philosophie afin de pouvoir reconnaître dans les propos des élèves des éléments de réponse déjà donnés par les philosophes. On voit ainsi qu'il ne s'agit pas de rejeter la tradition, au contraire, il s'agit plutôt de s'en servir comme support dans les discussions et surtout à la suite des discussions pour éclairer la recherche de la communauté par des réflexions qui ont été élaborées auparavant; les élèves pourront alors constater qu'on ne réinvente pas le bouton à quatre trous! Le recours à des questions 
traditionnelles permet de se laisser guider par des professionnels, ceux qui ont réfléchi une vie entière sur les questions philosophiques.

\section{La Philosophie pour les enfants dans les collèges}

Étudions maintenant l'application de ce programme au niveau collégial. Nous savons qu'une des difficultés des élèves repose sur le fait que pour eux, la philosophie, c'est toute une histoire qui est déconnectée de leur expérience. Mais la philosophie est aussi un savoir-faire. C'est évidemment cet aspect pratique qui est le plus susceptible d'intéresser les élèves, mais il ne doit ni être exploité au détriment de l'histoire de la philosophie, ni être utilisé pour relater des faits vécus personnels. La méthode de Lipman permet la rencontre entre l'histoire de la philosophie et le savoir-faire philosophique parce que la Philosophie pour les enfants c'est non seulement un savoir-faire, mais aussi l'histoire de la philosophie. Derrière chaque personnage des romans écrits par Lipman et son équipe nous pouvons trouver des philosophes types. Un des avantages de cette approche, c'est que l'élève est introduit non à une réponse philosophique d'un penseur démodé, mais aux questions qui furent celles de tous les philosophes et qui ont constitué le fil de l'histoire de la philosophie. On assiste alors à la rencontre du penser par et pour soi-même avec l'histoire de la philosophie, dans le dialogue philosophique. Et à bien des égards, le dialogue est un outil pédagogique plus puissant que la leçon magistrale, comme je tenterai de lillustrer. Ce serait donc un atout majeur de s'en servir dans les salles de classe.

Insérer la Philosophie pour les enfants au collégial permettrait donc d'atteindre notre objectif qui est d'initier les élèves autant à l'histoire de la philosophie qu'au dialogue philosophique. Pour ce qui est maintenant de la méthode à adopter pour appliquer la Philosophie pour les enfants à l'enseignement de la philosophie au collégial, elle reste essentiellement la même qu'avec les enfants, et se divise donc en trois parties : il s'agit d'abord de lire en commun, en table ronde, un extrait d'une œuvre philosophique. Par ailleurs, les histoires de Lipman s'adressant de prime abord à des enfants, nous pourrions quant à nous puiser nos lectures chez les plus grands auteurs de la philosophie, avec le Banquet de Platon par exemple ou encore inventer une histoire philosophique adaptée à leur réalité, comme il en existe déjà ${ }^{2}$. Le texte sert d'intermédiaire entre la culture philosophique et l'élève. En plus de développer le respect, cette méthode de travail permet à la classe de se transformer en commu- 
nauté de recherche, en incitant tous les membres à parler et à approfondir un thème dans une recherche commune. Enfin, le texte est la base sur laquelle s'érige le dialogue, il est le moteur d'une pensée qui s'apprête à naître.

La deuxième étape consiste à procéder à la cueillette des questions. Les élèves se sentent alors impliqués dans le processus pédagogique, puisqu'ils participent à l'établissement de l'ordre du jour en choisissant eux-mêmes le thème de la discussion. C'est cette période qui permet aux élèves de s'approprier le questionnement philosophique puisqu'il ne s'agit plus de répondre à une question imposée par le professeur, mais plutôt de réfléchir soi-même pour découvrir ses propres interrogations. Par ailleurs, cette étape qui semble si simple de prime abord nécessite une période d'adaptation pour les élèves. En effet, ils n'ont pratiquement jamais développé le réflexe de poser des questions et de s'interroger sur la matière; pour la majorité, ils sont à l'école pour écouter et ne pas déranger le professeur qui transmet le savoir. La cueillette des questions est donc un processus qui mérite beaucoup d'attention et peut-être même un entraînement pour qu'elle devienne comme une seconde nature chez l'élève. Le programme insiste d'ailleurs sur cette étape parce qu'elle rejoint l'essence de la philosophie; dès l'origine, la philosophie a débuté par un questionnement : il y a eu des philosophes parce que des gens se sont interrogés sur le monde dans lequel ils se trouvaient. Après ce premier pas vers la philosophie, il ne reste plus qu'à rendre fécond le questionnement des élèves.

Pour la troisième étape de la démarche, après que les élèves ont choisi démocratiquement la question qui les intéressait le plus, le professeur anime une discussion dans le cadre d'une communauté de recherche philosophique pendant laquelle il incite les élèves à l'utilisation des outils de la pensée. La démarche de la Philosophie pour les enfants au collégial permet aux élèves d'acquérir les outils de la pensée que j'ai énumérés plus tôt par l'encouragement du professeur qui les conduit à en utiliser plusieurs pendant les discussions après les avoir initiés à ces concepts. Ces outils sont par ailleurs déjà au programme des cours de philosophie, dans la portion consacrée à l'enseignement de la logique. J'utilise l'exemple de l'enseignement de la logique pour illustrer la pertinence de transformer un enseignement magistral en communauté de recherche avec les élèves. Traditionnellement, le professeur explique magistralement ce que sont

2. Gilbert Talbolt, La découverte de Phil et Sophie, 1993. 
les outils de la dialectique tel qu'Aristote les a présentés, des syllogismes aux sophismes, en passant par le principe de noncontradiction. Or, de cette façon, l'élève a beaucoup moins de chances de retenir quoi que ce soit, et encore moins de l'utiliser, puisqu'on lui enseigne les outils de la pensée comme s'il s'agissait de théorèmes mathématiques abstraits; si on veut construire une maison, la théorie devient insuffisante, il faut savoir manier le marteau. En fait, l'élève ne s'imagine même pas qu'il peut se servir quotidiennement des instruments dialectiques d'Aristote. Avec la méthode de la pratique de la philosophie, les élèves sont non seulement amenés à réfléchir sur ces concepts, mais aussi à les utiliser spontanément dans une discussion philosophique. C'est ici que la philosophie deviendrait utile à leurs yeux. Je crois que les élèves pourraient atteindre plus facilement les objectifs ministériels en mettant les concepts en pratique.

L'enjeu du dialogue est de faire advenir l'art de penser. En ce sens, le rôle du professeur est de s'assurer que le questionnement initial aboutit bel et bien à un dialogue où les élèves apprennent à penser par et pour eux-mêmes. L'aspect créatif de la pensée autonome ne peut cependant pas être enseigné comme on enseigne une vérité mathématique. Mais pour Lipman, il est possible d'atteindre un objectif que l'on ne peut enseigner. Ainsi, la créativité propre à la pensée autonome peut être encouragée par les professeurs qui peuvent doter les élèves d'une force inventive. Pour reprendre un vieux modèle, Socrate avait déjà cette attitude; il était accoucheur d'idées. Les professeurs du collégial peuvent reprendre ce rôle de la manière suivante : en suivant le débat où il mène au lieu d'imposer son propre cheminement réflexif, en interrogeant les élèves sur le sens des mots qu'ils utilisent et sur l'ensemble des outils de la pensée auxquels ils ont recours, bref en accompagnant les élèves dans leur réflexion. Et je répète qu'il ne s'agit pas de faire comme s'il n'y avait pas de tradition philosophique ; au contraire, celle-ci est au cœur de la démarche des élèves parce que la tradition, c'est un ensemble de questions que des hommes se sont posées, et c'est aussi une pratique de la philosophie.

\section{Est-ce réalisable?}

Certains professeurs du collégial doutent de la faisabilité d'un tel projet. Soit. Voyons le principal argument qui peut être avancé contre l'insertion du programme de Philosophie pour les enfants au collégial. Les objectifs du ministère sont si serrés qu'il semble impossible pour 
un professeur de couper ses heures d'enseignement pour laisser place au dialogue. II semble que pour permettre aux élèves d'atteindre les objectifs, "le plus d'heures possibles d'enseignement théorique" est encore insuffisant. J'ai une double réaction face à cette attitude. Premièrement, je ne crois pas que ce soit la bonne manière d'envisager l'insertion du programme dans les cours. Cette attitude reflète la croyance que l'enseignement de la philosophie doit être distinct de l'enseignement du savoir-faire philosophique. Or, le fait de prendre du temps pour discuter en classe ne doit pas être vu comme du temps perdu. II s'agit, selon moi, d'une variante qui permet aussi d'atteindre les objectifs ministériels, et surtout qui permet d'initier les élèves à l'art de philosopher, indispensable dans la formation d'un citoyen. Deuxièmement, le manque de temps envisagé par quelquesuns est quelque peu dramatisé! II ne s'agit pas de discuter chaque cours et tout le cours, mais plutôt de doser les deux types d'enseignement afin que les élèves soient initiés non seulement de manière rigoureuse à l'histoire de la philosophie, mais aussi de manière pratique, appliquée, à la réflexion philosophique. II pourrait suffire de discuter (dans le cadre d'une communauté de recherche philosophique encadrée par le professeur dans le but d'utiliser les outils de la pensée) une heure au début de quelques cours, ou lors de l'introduction à un nouvel objectif ministériel, ou enfin aussi souvent que le juge bon le professeur, en autant que l'une des deux manières d'enseigner la philosophie ne prenne pas tout l'espace aux dépens de l'autre. II faut noter cependant que les bénéfices d'une telle approche ne peuvent se faire sentir en deçà d'un certain nombre d'heures de pratique.

\section{Conclusion}

J'aimerais m'attarder avant de terminer sur un des effets positifs de l'implantation de la méthode de Philosophie pour les enfants au collégial, qui repose sur l'union de la forme et de la matière ou de la pédagogie et de la matière enseignée. Cette démarche pourrait éliminer les effets secondaires d'une approche purement théorique par la tradition philosophique aussi bien que d'une approche purement pratique où les élèves ne font qu'exprimer leurs opinions. Ainsi, la philosophie retrouverait peut-être sa véritable essence.

Évidemment, je ne rejette pas indistinctement tout le travail qui a été fait depuis la création des collèges publics québécois, ni ne propose de remplacer le devis ministériel par quelque chose de totalement différent et neuf. Au contraire, les cours de philosophie du 
collégial, tels qu'ils sont présentement dans la grande majorité, sont indispensables pour la formation des élèves. Ce que je propose, c'est une méthode d'approche, une façon de parvenir à transmettre le contenu déjà élaboré dans les devis ministériels. S'il est vrai qu'un esprit sans structure n'a pas les capacités minimales à l'intégration de sa formation philosophique, à quoi sert-il d'enseigner l'histoire de la philosophie si on ne permet pas aussi aux élèves de développer un esprit structuré en leur donnant les outils? Selon moi, je le répète, les deux parties de l'apprentissage, enseigner la philosophie et apprendre aux élèves à philosopher, sont indispensables à la formation philosophique des élèves; la vertu est au milieu.

Si je propose cette approche comme une solution possible au problème de la philosophie au collégial, ce n'est pas parce que je crois qu'aucun professeur n'utilise cette approche; je sais que plusieurs professeurs ont inséré la méthode Lipman dans leurs cours (et même avant que celui-ci ait élaboré son programme). Je dis seulement que si tant d'élèves continuent à avoir une mauvaise opinion de leurs cours de philosophie, c'est que le programme gouvernemental permet dans ses objectifs qu'un professeur passe outre cette composante du dialogue selon moi cruciale dans l'apprentissage de la philosophie. Je ne prétends donc pas réinventer l'enseignement de la philosophie; je souhaite simplement susciter une réflexion sur l'avenir de la philosophie au collégial, réflexion orientée vers une méthode.

Certains me reprocheront peut-être mon enthousiasme naïf de débutante, mais je crois que c'est une qualité. Je suis émerveillée lorsque j'entends des jeunes discuter sur des thèmes fondamentaux de l'expérience humaine, des thèmes philosophiques. Ce sont des personnes libres. Libres de s'émerveiller, de réfléchir, de s'exprimer et d'argumenter. Nous pouvons créer des conditions pour que la liberté se déploie. Un philosophe a déjà dit, il y a bien longtemps, que l'étonnement est le premier moteur de la philosophie. Si nous pouvons réussir à le transmettre, ce fameux taumazein philosophique, alors la société sera composée de meilleurs citoyens ${ }^{3}$.

\section{Marie-Georges Bélanger Université Laval}

3. Je remercie Michel Sasseville pour ses précieux conseils. 


\section{BIBLIOGRAPHIE*}

Ouvrages généraux francophones sur la pratique de la philosophie avec les enfants :

Caron, Anita (dir.). Philosophie et pensée, chez l'enfant. Montréal, Les éditions Agence d'ARC, 1990.

Daniel, Marie-France. La philosophie et les enfants. Montréal, Les éditions logiques, 1992.

Marcil-Lacoste, Louise. La philosophie pour enfants. L'expérience Lipman. Sainte-Foy, Les éditions Le Griffon d'argile, 1990.

Sasseville, Michel (dir.). La pratique de la philosophie avec les enfants. Sainte-Foy, Les Presses de l'Université Laval, 1999.

\section{Matériel de base de la méthode de philosophie pour les enfants, écrit par Matthew Lipman et son équipe, traduit en français :}

Lipman, Matthew. Pixie. Trad. Arsène Richard. Moncton, Les éditions d'Acadie, 1984.

Kio et Augustine. Trad. Arsène Richard. Moncton, Les éditions d'Acadie, 1987.

La découverte de Harry. Trad. et adaptation par Michel Haguette. Montréal, La commission des écoles catholiques de Montréal, Service des études, 1987.

Guide d'accompagnement de "La découverte de Harry». Trad. MarieMarthe Ménard-Markiza, 1982. Révisé par Jean Laberge. Montréal, Université du Québec à Montréal, 1991.

Talbot, Gilbert. La découverte de Phil et Sophie. Adaptation de Harry Stottlemeier's Discovery de Lipman. Préface de Lipman. Québec, Éditions du Loup de Gouttière, 1993.

- Cette bibliographie n'est évidemment pas complète, elle n'est qu'à titre indicatif pour donner une piste de recherche à ceux qui souhaitent approfondir la pratique de la philosophie avec les enfants. 